Radial and Nonradial Pulsations as Probes of Stellar Physics

ASP Conference Series, Vol. 259, 2002

C. Aerts, T.R. Bedding, \& J. Christensen-Dalsgaard, eds.

\title{
Evolution of Am and Ap Stars
}

\author{
O. Richard ${ }^{1}$, G. Michaud ${ }^{1}$, J. Richer ${ }^{1}$ \\ Département de physique, Université de Montréal, Montréal, H3C 3J7, \\ Canada
}

\section{The models}

This work is a continuation of the Montréal stellar evolutionary model development (Richard et al., 2001 and references therein) where the effects of atomic diffusion are taken into account for 28 elements (see Turcotte, these proceedings), along with an approximate treatment of turbulent transport (Richer et al., 2000).

We used the CEFF equation of state and the Bahcall nuclear energy generation routine. The Rosseland opacity is computed at each time step, from the OPAL monochromatic opacities for 21 elements (to which we added locally computed spectrum tables for $\mathrm{Li}, \mathrm{Be}$, and $\mathrm{B}$ ) for each mesh point in the model for the current local chemical composition. Convection and semi-convection are taken into account in every species' diffusion velocity and in the determination of the thermal gradient (Richard et al., 2001). The models also take into account gravitational settling, thermal diffusion, radiative accelerations and turbulent transport. The radiative accelerations are from Richer et al. (1998). The turbulent transport is modeled as a diffusion process (Richer et al., 2000) with diffusion coefficient: $D_{\mathrm{T}}=\omega D(\mathrm{He})_{0}\left(\rho / \rho_{0}\right)^{n}$ where $D(\mathrm{He})_{0}$ is the He atomic diffusion coefficient at density $\rho_{0}$.

All models shown here were assumed homogeneous on the PMS, with the abundance mix defined in Table 1 of Turcotte et al. (1998). The $1.7 M_{\odot}$ models have been computed up to $230 \mathrm{Myr}$, in about 300 time steps. Each model has about 1500 layers. The 3 series of models use different assumptions about turbulent transport between the surface and the Fe convective zone, as well as under the Fe convective zone. Am models: these models, named "1.70T5.3D1K-3", have enough turbulence to mix the layers from the surface down to $\log \left(\Delta M / M_{*}\right) \simeq$ -5, and could reproduce the observed AmFm abundance anomalies (see Fig. 20 of Richer et al., 2000). "T5.3D1K-3" means that $D_{\mathrm{T}}$ is equal to $D_{\mathrm{MLT}}$ for $\log T<5.3$, to 1000 times the He atomic diffusion coefficient at a reference density $\rho_{0}=\rho(\log T=5.3)$, and decreases as $\rho^{-3}$ for $\log T \geq 5.3$. Ap models: these models, named "1.70r1M-3" and "1.70T5.3D5-3", have less turbulence than the Am models and show more pronounced abundance anomalies. "r1M- 3 " means that $D_{\mathrm{T}}$ is equal to $10^{6}$ times the He atomic diffusion coefficient at a reference density $\left(\rho_{0}=8 \times 10^{-6} \mathrm{~g} \mathrm{~cm}^{-3}\right)$, is proportional to $\rho^{-3}$, and extends from the bottom of the topmost convective zone to the center of the model.

\footnotetext{
${ }^{1}$ CEntre de Recherche en Calcul Appliqué (CERCA), 5160 boul. Décarie, bureau 400,
} Montréal, PQ, Canada, H3X 2H9 


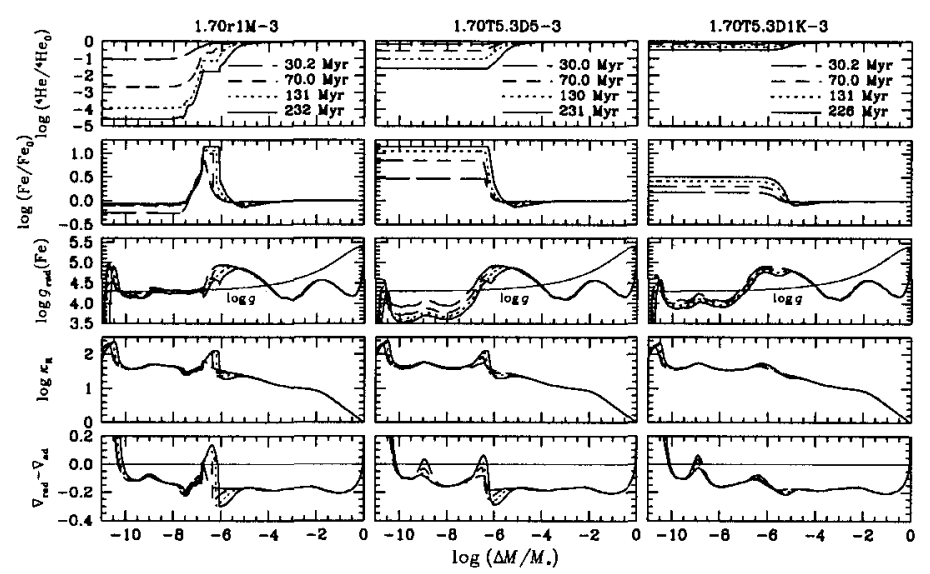

Figure 1. Effect of turbulence on the structure and composition of the models at few ages.

\section{Results}

In the 3 models, the Fe abundance between $\log \left(\Delta M / M_{*}\right) \simeq-5$ and -4 decreases as $\mathrm{Fe}$ is pushed by radiative pressure toward the surface (see Fig. 1). As the 1.70T5.3D5-3 and 1.70D1K-3 models are fully mixed between $\log T=5.3$ and the surface, the Fe surface abundances increase. For the $1.70 \mathrm{r} 1 \mathrm{M}-3$ model, $\mathrm{Fe}$ is also supported by radiative pressure between $\log \left(\Delta M / M_{*}\right) \simeq-5$ and -7 but is not supported higher up. The $\log \kappa_{\mathrm{R}}$ and $\nabla_{\text {rad }}-\nabla_{\text {ad }}$ curves show clearly that the $\mathrm{Fe}$ accumulation causes an increase of the opacity and generates a convective zone in the $1.70 \mathrm{r} 1 \mathrm{M}-3$ and $1.70 \mathrm{~T} 5.3 \mathrm{D} 5-3$ models. In the $1.70 \mathrm{r} 1 \mathrm{M}-3$ model, He is reduced by $\times 10^{-5}$ between the surface and the Fe convective zone; under the $\mathrm{Fe}$ convective zone the helium profile is similar to that of the 1.70T5.3D5-3 model. In the 1.70D1K-3 model, turbulence is large enough to strongly oppose helium diffusion. The abundance anomalies caused by diffusion extend over $\sim 20 \%$ of the radius for some species. They are not as superficial as is often believed. Only the region between $0.5<r / R_{*}<0.8$ retains the original composition of the star. A preliminary study of the models' pulsation properties is presented in Michaud et al. (these proceedings).

\section{References}

Richard, O., Michaud, G., \& Richer, J. 2001, ApJ, 558, 377

Richer, J., Michaud, G., Rogers, F., Iglesias, C., Turcotte, S., \& LeBlanc, F. 1998, ApJ, 492, 833

Richer, J., Michaud, G., \& Turcotte, S. 2000, ApJ, 529, 338

Turcotte, S., Richer, J., Michaud, G., Iglesias, C.A., \& Rogers, F.J. 1998, ApJ, 504,539 